Gabriela de Oliveira Silva ${ }^{1}$ Flávia Tavares Silva Elias ${ }^{2}$

${ }^{1}$ Mestra em Políticas Públicas em Saúde. Escola Fiocruz de Governo. Fundação Oswaldo Cruz. Brasília/DF. Brasil. E-mail: gabioliveira18@gmail.com

${ }^{2}$ Pesquisadora do Programa de Evidências em Políticas e Tecnologias em Saúde/Fundação Oswaldo Cruz. Brasília/ DF. Brasil. E-mail: flavia.elias@fiocruz.br

Correspondência:

Gabriela de Oliveira Silva.

Avenida L3 Norte, s/n, Campus Universitário Darcy Ribeiro, Gleba A. CEP: 70.904-130. Brasilia - DF. Brasil. Telefone:

(31)986895716. Email: gabioliveira18@ gmail.com

Este artigo compõe um dos subcapítulos de resultados da dissertação de mestrado da primeira autora, orientada pela segunda autora, intitulada "Parcerias para o Desenvolvimento Produtivo e a produção pública de medicamentos: uma proposta de monitoramento estratégico", 2017, Escola Fiocruz de Governo, Brasília-DF, Brasil.

\section{Parcerias para o desenvolvimento produtivo: um estudo de avaliabilidade}

\section{Productive development partnerships: an availability study}

\section{RESUMO}

Introdução: As Parcerias para o Desenvolvimento Produtivo (PDP) são uma das iniciativas do Ministério da Saúde de fomento ao desenvolvimento do Complexo Econômico Industrial da Saúde para ampliação do acesso a medicamentos à população. Entretanto, ainda não se verifica uma avaliação sistematizada das PDP, de seus resultados e impactos. O estudo de avaliabilidade permite verificar se as PDP estão prontas para serem avaliadas de forma útil e sistematizada, bem como estruturar um programa de avaliação.

Objetivo: Apresentar um estudo de avaliabilidade das PDP e propor indicadores para monitoramento e avaliação.

Método: coleta e análise de documentos normativos e da literatura publicada, montagem e validação dos modelos lógicos do problema, dos componentes operacionais e estratégicos da iniciativa e construção de indicadores.

Resultados: Os três modelos lógicos construídos demonstraram que as ações e atividades estão organizadas de modo a gerar produtos e resultados. Há objetivos definidos, beneficiários e público alvo. Foram propostos 14 indicadores, sendo sete relacionados ao monitoramento das fases de execução do projeto (fases I, de submissão e análise da proposta; II, de implementação do projeto; III, de transferência de tecnologia; e IV, de internalização de tecnologia), seis à avaliação dos resultados intermediários da transferência de tecnologia e um do resultado final referente à ampliação do acesso aos produtos objetos de PDP. Conclusão: Verificou-se que a iniciativa das PDP se encontra normatizada de modo a permitir a realização de avaliações sistematizadas por meio dos modelos e indicadores propostos.

Palavras-chave: Avaliação de Programas e Projetos de Saúde. Indicadores. Transferência de Tecnologia. 


\begin{abstract}
Introduction: The Productive Development Partnerships (PDP) are one of the Brazil's Ministry of Health initiative for wide population access to medicines and for the development of the economic industrial health care complex. However, there is still no systematic evaluation of the PDP, its results and impacts. The availability study allows verifying that the PDP are ready to be evaluated in a useful and systematized way, as well as to structure an evaluation program.
\end{abstract}

Objective: present an availability study on this initiative and suggest indicators for monitoring and assessment.

Method: gathering and analysis of normative documents and published literature, assembly and validation of logic models of the problem, of the operational and strategic components, and construction of indicators.

Results: The three built logic models showed that the actions and activities are organized so that products and results are generate. There are defined objectives, recipients and users. Based on logic models, fourteen indicators were proposed, seven related to the monitoring of the execution stages of the project (phase I, proposal submission and analysis; II, project implementation; III, technology transfer and absorption; and IV, technology internalization), six to the evaluation of the technology-transfer intermediate results and one to the final result regarding the widening of the access to the products subjected to PDP.

Conclusion: It was verified that the PDP initiative is regulated to enable the fulfillment of systematized assessments through proposed models and indicators.

Keywords: Evaluation of Health Programs and Projects. Indicators. Technology Transfer.

\section{INTRODUÇÃO}

As Parcerias para o Desenvolvimento Produtivo (PDP) são uma das iniciativas do Estado brasileiro de utilização do poder de compra do setor saúde para a execução de projetos que associam o acesso a medicamentos de qualidade ao desenvolvimento do Complexo Econômico Industrial da Saúde (CEIS), o conjunto articulado de setores industriais e prestadores de serviços que agregam as dimensões econômica e social da saúde ${ }^{1}$. Tal iniciativa é coordenada pelo Ministério da Saúde (MS) e foi lançada em 2009 na reunião do Grupo Executivo do Complexo Industrial da Saúde (GECIS).
Trata-se de uma estratégia que fortalece o modelo de economia racional desenvolvimentista, tendo como meta o aumento da capacidade tecnológica e produtiva industrial, com a participação ativa do Estado por meio da coordenação da iniciativa e da absorção de plataformas para fabricação efetiva de tecnologias estratégicas pelos produtores públicos para atendimento das demandas do Sistema Único de Saúde (SUS).

Cassiolato e Szapiro² apontam que essa iniciativa tem gerado resultados significativos, contando com a participação do capital estrangeiro na produção de insumos para o SUS. Andrade e Perini ${ }^{3}$ e Domingues et al. $^{4}$ apresentam como 
resultados da iniciativa: a incorporação de novas plataformas produtivas nas instituições públicas; a economia nos gastos do MS na medida de redução anual de preços pelos produtores; a perspectiva de diminuição do déficit da balança comercial do Brasil no setor saúde devido à redução da importação de insumos ativos e medicamentos; a redução da vulnerabilidade do SUS em relação a medicamentos de alto valor agregado e complexidade tecnológica, sendo possível contribuir para a ampliação do acesso da população a produtos estratégicos.

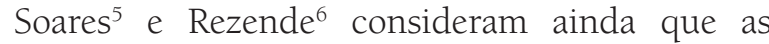
PDP possibilitam uma ampliação do portfólio de produtos, dos nichos de atuação e do faturamento dos produtores públicos, fortalecendo-os e ampliando seu papel na regulação do mercado.

Segundo Golgher et al. ${ }^{7}$, apesar de ser cedo para mensurar o impacto das PDP no SUS, a estratégia possui potencial de se tornar um grande marco. Para tanto, o controle, o monitoramento e a avaliação da estratégia são indispensáveis para que se atinjam os resultados almejados da iniciativa ${ }^{8} \mathrm{e}$ devem ser postos em prática de modo a verificar a eficiência da transferência da tecnologia nos projetos e evitar que a finalidade das parcerias seja desvirtuada9.

Entretanto, ainda não se verifica uma avaliação sistematizada das PDP, de seus resultados e impactos. A construção e o aprimoramento das normativas dessa iniciativa ocorreram na medida de sua implantação. A primeira normativa específica foi publicada três anos depois do lançamento da estratégia, a Portaria $n^{\circ} 837 / 2012$, e esta foi revisada em 2014 diante das necessidades verificadas pela governança e das recomendações dos órgãos de controle, gerando a Portaria no 2.531/2014.

Neste sentido, um estudo de avaliabilidade pode trazer a compreensão da iniciativa implementada e auxiliar na identificação dos elementos úteis e viáveis para análise, bem como possibilitar o desenho de uma avaliação adequada ${ }^{10}$. Entende-se por estudo de avaliabilidade a análise da medida em que iniciativas estão prontas para serem avaliadas de forma útil e sistematizada ${ }^{10}$.

O modelo lógico é uma ferramenta útil para realizar esse tipo de estudo e tem sido utilizado pelo Ministério do Planejamento, Orçamento e Gestão para aperfeiçoar o desenho de programas do Plano Plurianual (PPA). Segundo Cassiolato e Gueresi ${ }^{11}$, o modelo lógico é um instrumento que permite organizar as ações de um programa ou política interligando-as aos resultados esperados, servindo "como um organizador para desenhar avaliação e medidas de desempenho, focalizando nos elementos constitutivos do programa e identificando quais questões devem ser colocadas para avaliação e quais medidas de desempenho são relevantes". Embora seja usualmente empregado na avaliação ex-ante de programas, a sua aplicação ex-post também tem sido realizada ${ }^{11}$.

Assim, este artigo apresenta um estudo de avaliabilidade das PDP, elaborado por meio de modelos lógicos sobre o problema que as PDP buscam enfrentar, os componentes estratégicos e operacionais da iniciativa. Esse estudo tem o propósito de ampliar a compreensão sobre o desenho dessa estratégia e promover a estruturação de um processo avaliativo para o seu aprimoramento.

\section{MÉTODO}

Este estudo fundamentou-se na abordagem qualitativa e na adaptação da metodologia apresentada pelo Instituto de Pesquisa Econômica Aplicada (IPEA) em Nota Técnica de Cassiolato e Gueresi $^{11}$.

Primeiramente, foi realizada busca e análise de normativas e publicações referentes às PDP no portal do MS (www.saude.gov.br/deciis), no Google Acadêmico e na Biblioteca Virtual em Saúde. Para tal, foram utilizadas as seguintes questões: (1) qual o problema que as PDP se propõem a enfrentar?; (2) quais suas principais consequências?; (3) quais as causas mais importantes?; (4) qual o objetivo, o públicoalvo e os beneficiários das PDP?; (5) quais ações compõem as PDP e a finalidade destas?; (6) quais os produtos previstos para cada ação?; (7) quais os resultados esperados e quais se pretende alcançar no período do PPA?; (8) como as ações e produtos contribuem para alcançar os resultados?; e (9) quais os fatores de contexto que podem afetar o desempenho das PDP?

O resultado desta análise orientou a construção, por meio de desenhos no Microsoft Power Point, de três modelos lógicos que têm como base o Planejamento Estratégico Situacional (PES): (a) explicação do problema; (b) referências básicas das PDP; e (c) estruturação das PDP para alcance dos resultados; fundamentados nas respostas das questões 1 a 3, 4, e 5 a 9 , respectivamente. O terceiro 
modelo foi composto pelos elementos da estrutura lógica (recursos, ações, produtos, resultados intermediários e final e efeitos) associados às fases do processo de estabelecimento das parcerias e às dimensões de gestão do Octógono da Inovação (estratégia, liderança, relacionamento, cultura, pessoas, processos, estrutura e funding $)^{12}$.

O Octógono da Inovação, um instrumento proprietário da Innoscience - Consultoria de Gestão da Inovação ${ }^{12}$, foi utilizado para subsidiar a construção deste terceiro modelo uma vez que as PDP são consideradas uma iniciativa inovadora na gestão pública federal.

Os modelos construídos foram apresentados a três gestores e pesquisadores com experiência na temática das PDP, que discutiram as adequações $\mathrm{e}$ as inclusões pertinentes. Para validação do terceiro modelo, realizou-se também o teste de consistência e a análise de vulnerabilidade.

O teste de consistência compreendeu a análise de hipóteses sobre a estruturação das PDP para alcance dos resultados esperados. Para as ações desse modelo, foram feitas apostas na forma de "se ... então" articulado com os produtos, resultados intermediários e final ${ }^{11}$.

$\mathrm{Na}$ análise de vulnerabilidade, foram verificadas eventuais fragilidades nessas apostas, ou seja, nas ações para alcance dos resultados. Tal análise foi realizada a partir da identificação de condições de invalidação de cada aposta, da verificação da probabilidade de ocorrerem e do impacto gerado se ocorressem ${ }^{11}$. A probabilidade e o impacto foram graduados, na percepção dos autores, em uma escala simples de alto, médio e baixo. Identificada vulnerabilidade a uma condição de invalidação, foram definidas ações preventivas ou de controle.

Em seguida, foram elaborados indicadores de monitoramento e avaliação das PDP, e caracterizados pela sua denominação, conceito, método de cálculo e fontes de verificação, conforme adaptação do modelo utilizado pela Rede Interagencial de Informações para a Saúde segundo os "Indicadores básicos para a saúde no Brasil: conceitos e aplicações" de 2008 da Organização Pan-Americana da Saúde.

\section{RESULTADOS E DISCUSSÃO}

Na busca documental realizada, não se identificou a construção ex-ante pelo MS da árvore de problemas, da matriz lógica e do modelo lógico relacionado às PDP. A elaboração ex-post do modelo lógico de explicação do problema auxiliou na visualização dos elementos ensejadores de criação das PDP, possibilitando identificar o problema principal e seus descritores e verificar a interação entre as suas causas e consequências (Figura 1).

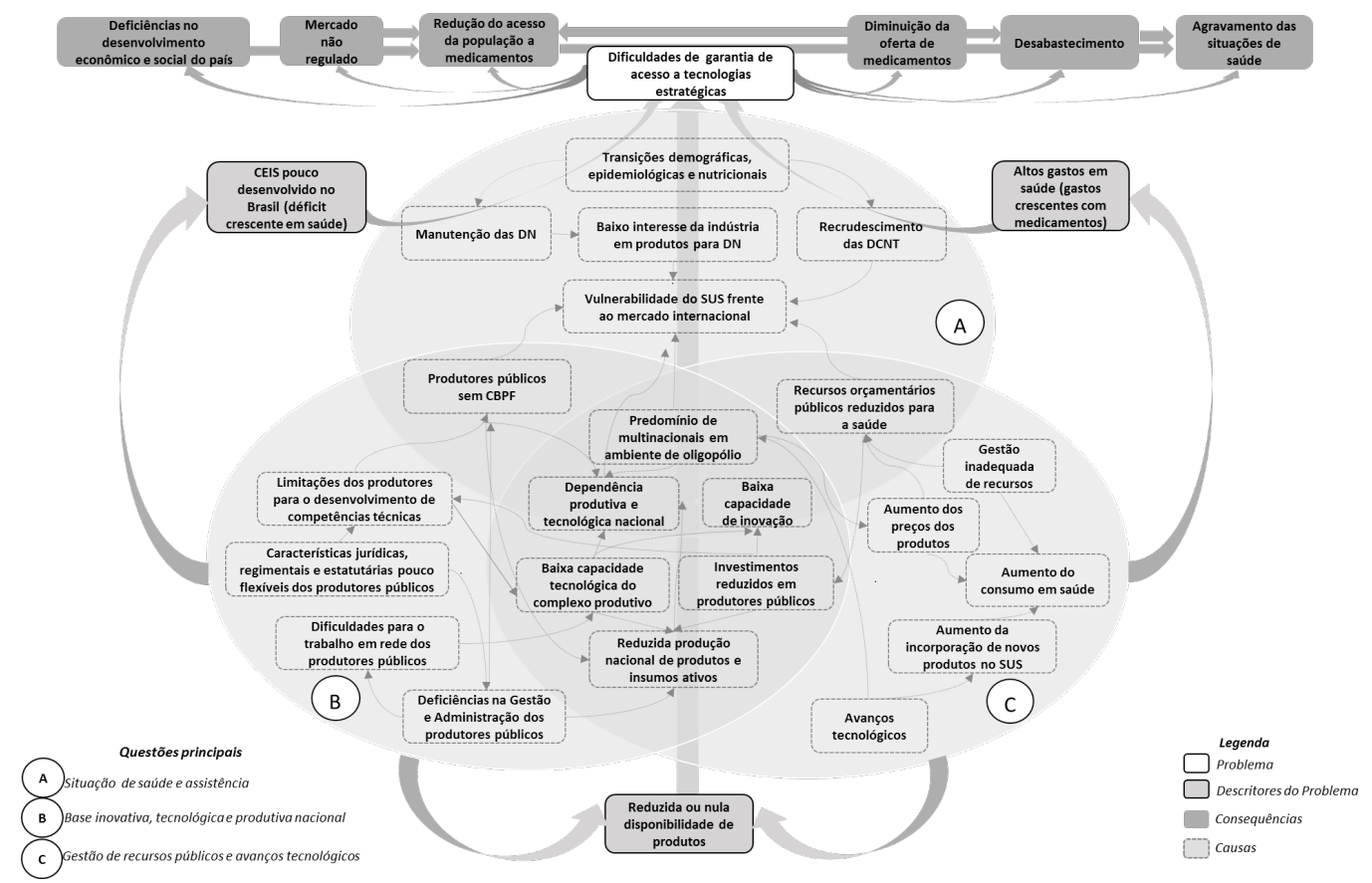

Figura 1

Modelo lógico de explicação do problema que as Parcerias para o Desenvolvimento Produtivo buscam enfrentar 
O problema central que as PDP buscam enfrentar refere-se às dificuldades de garantia de acesso a tecnologias estratégicas. Este é talvez o desafio mais complexo a ser enfrentado pelos setores público e privado e organizações não governamentais envolvidos na oferta de medicamentos ${ }^{13}$. Para dar precisão a esse problema e demonstrar a sua dimensão, identificam-se três descritores principais: (1) altos gastos em saúde: somente em 2015, as transferências diretas do Fundo Nacional de Saúde e fundo a fundo para Estados e Municípios atingiram cerca de $\mathrm{R} \$ 73,6$ bilhões $^{14}$, o que representa um aumento de 79,3\% em relação a 2009; (2) CEIS pouco desenvolvido no Brasil: déficit da balança comercial em saúde mostra-se expressivo e crescente, sendo que, em 2009, era de US $\$ 7,2$ bilhões e atingiu o patamar de US\$ 11,5 bilhões em $2014^{15}$; especificamente na cadeia produtiva farmacêutica, o déficit teve aumento de US $\$ 4,8$ bilhões de 2009 a $2014^{16}$; e (3) reduzida ou nula disponibilidade de alguns produtos essenciais, apesar dos crescentes investimentos na estratégicas está relacionado a múltiplas causas, que podem ser associadas a três questões principais que muitas vezes se sobrepõem: (a) situação de saúde e assistência; (b) base inovativa, tecnológica e produtiva nacional; e (c) gestão de recursos públicos e avanços tecnológicos.

Quanto às consequências do problema, estão relacionadas a fatores que interferem na sustentabilidade dos sistemas de saúde: deficiências do desenvolvimento econômico e social, regulação do mercado, desabastecimento, redução da oferta e do acesso da população a medicamentos e o agravamento das situações de saúde.

No segundo modelo lógico, é apresentado o que foi feito para solucionar esse problema, ou seja, a formulação da estratégia, as referências básicas das PDP, apresentando o problema e seus descritores, os objetivos, o público-alvo e os beneficiários da iniciativa (Figura 2).

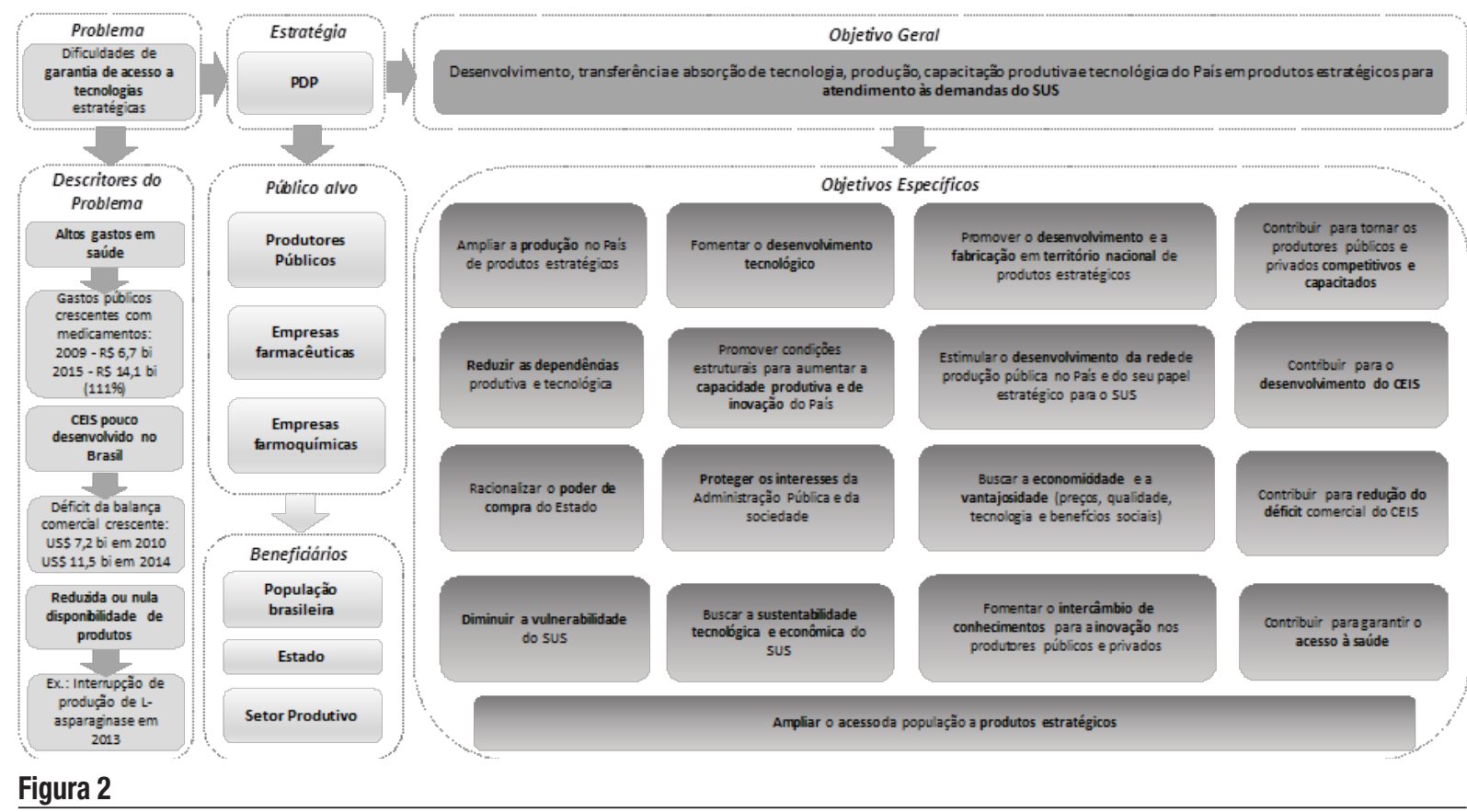

Modelo lógico das referências básicas das Parcerias para o Desenvolvimento Produtivo

assistência farmacêutica: como exemplo, cita-se o desabastecimento em 2013 da L-asparaginase, usada no tratamento pediátrico da leucemia linfoide aguda e de linfoma não Hodgkin, como evidenciado na prática profissional da primeira autora no MS.

Como demonstrado na Figura 1, esse problema de falta de garantia de acesso a tecnologias
Os objetivos das PDP estão relacionados principalmente à ampliação de acesso da população a produtos estratégicos, ao desenvolvimento do CEIS no Brasil e à economicidade para a Administração Pública.

Para alcance desses objetivos, tem-se como público-alvo os atores relacionados à cadeia produtiva farmacêutica: os produtores públicos, 
responsáveis pela absorção de tecnologia e fornecimento de medicamentos ao MS, as empresas farmacêuticas capacitadas para a transferência de tecnologia e as farmoquímicas envolvidas no desenvolvimento dos insumos ativos no Brasil.

Como beneficiários dessa estratégia, identificam-se o Estado brasileiro, ao absorver a tecnologia, poder empregá-la, disseminá-la e obter o medicamento a preços menores; o setor produtivo, ao fornecer o produto em larga escala e poder se desenvolver; e a população brasileira, ao ter assegurado o abastecimento do SUS ${ }^{18}$.

O terceiro modelo lógico construído refere-se à estruturação da iniciativa para alcance dos resultados e demonstra como fazer para a iniciativa das PDP funcionar. Esse modelo é composto por oito componentes que explicam a teoria das PDP e são úteis para nortear os processos de avaliação e aprimoramento da estratégia (Figura 3). das parcerias; (c) pessoas e cultura perpassam todas as fases e ações por serem o instrumento de execução das PDP; (d) relacionamento e liderança estão envolvidos desde a articulação dos parceiros para elaboração da proposta até a finalização da parceria; (e) estratégia referese às fases iniciais, nas quais os gestores do MS definem a lista de produtos estratégicos, e a alta administração dos produtores públicos e privados identificam, nessa lista, os produtos que mais se adequam ao direcionamento das empresas para o estabelecimento de um projeto; e (f) funding que trata dos investimentos necessários para a execução dos projetos, tais como aquisição de equipamentos e adequação fabril, utilizando os recursos do fornecimento do produto ao SUS.

Os recursos envolvidos nas PDP são o segundo componente do modelo lógico. Nas fases iniciais de planejamento e negociação da parceria são, em sua maioria, não orçamentários. A partir da implementação do projeto e desenvolvimento

\section{Figura 3}

Modelo lógico de estruturação das Parcerias para o Desenvolvimento Produtivo para alcance dos resultados.

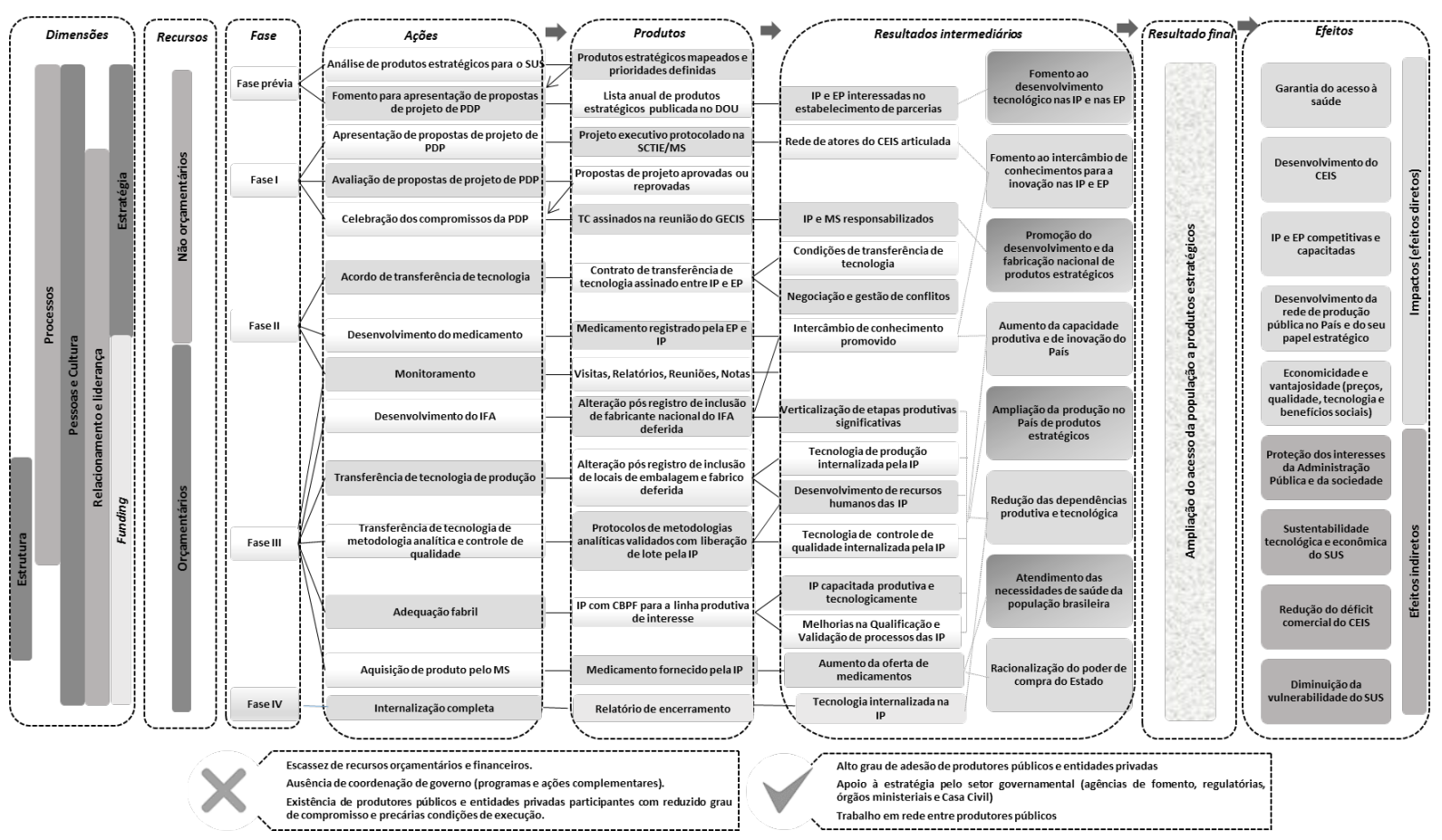

No primeiro componente desse modelo, está assinalada a maior relevância das dimensões do Octógono da Inovação em cada parte do processo de gestão das PDP: (a) estrutura refere-se à adequação dos produtores públicos e privados para as ações de transferência e absorção de tecnologia; (b) processos está relacionado às ações de análise dos projetos e de gestão e monitoramento do produto, são orçamentários visto que a sustentabilidade das parcerias está associada ao uso do poder de compra do setor saúde.

As cinco fases em que as PDP foram estruturadas representam o terceiro componente do modelo lógico, as quais incluem uma série de ações interdependentes e consecutivas que estão 
apresentadas no quarto elemento do modelo: fase prévia - definição da lista de tecnologias estratégicas pelo MS, com o apoio do GECIS, e fomento à apresentação de propostas; fase I submissão da proposta, análise pelas instâncias de avaliação e, em caso de aprovação, celebração do termo de compromisso entre o MS e o produtor público; fase II - implementação do projeto; fase III - transferência e absorção da tecnologia de forma efetiva e celebração do contrato de aquisição do produto entre o MS e o produtor público; e fase IV - internalização de tecnologia em condições de produção no Brasil e de portabilidade tecnológica pelo produtor público ${ }^{17}$. Por todas essas fases, perpassam as ações de monitoramento realizadas pelo MS e pela Agência Nacional de Vigilância Sanitária (ANVISA) no âmbito dos Comitês Técnico Regulatórios.

Todas essas ações visam alterar as causas críticas do problema e geram produtos ${ }^{11}$ aos beneficiários das parcerias. Tais produtos estão indicados no quinto componente do modelo e incluem estruturas e processos organizados nos produtores públicos, medicamentos desenvolvidos e disponibilizados com qualidade e segurança garantidas.

Em decorrência desses produtos, os resultados intermediários, sexto componente do modelo, refletem mudanças nas causas do problema nos aspectos econômicos e sociais, tais como: melhor gestão dos recursos públicos, maior capacitação produtiva e tecnológica nacional e aumento da oferta de produtos estratégicos.

Tais resultados conduzem ao sétimo elemento do modelo: ao resultado final esperado, objetivo macro das PDP, que reflete a mudança no problema, ou seja, a ampliação do acesso da população a produtos estratégicos. Tanto os resultados intermediários quanto o final estão associados aos objetivos da iniciativa, representados no modelo lógico das referências básicas das PDP, e a quatro metas do PPA 2016-2019, quais sejam: (a) reduzir, anualmente, o preço médio das aquisições de PDP; (b) ampliar de uma para nove as internalizações de tecnologias no SUS por PDP; (c) iniciar pelo menos quatro projetos de parcerias de pesquisa, desenvolvimento e inovação; e (d) desenvolver ou absorver, através de PDP, oito novos medicamentos ${ }^{19}$.

Há ainda os efeitos decorrentes dos resultados que estão apresentados no oitavo componente do modelo e que podem estar diretamente relacionados a eles (impactos), tais como garantia do direito fundamental à saúde e o desenvolvimento do CEIS; ou ser efeitos indiretos, como a diminuição do déficit da balança comercial em saúde e da vulnerabilidade do SUS.

O último componente do modelo refere-se aos fatores relevantes de contexto que podem favorecer o desenvolvimento das ações (fatores favoráveis), como o apoio e trabalho em rede dos setores produtivo e governamental, ou interferir negativamente, comprometendo-as (fatores desfavoráveis), como a restrição orçamentária e a falta de compromisso dos executores da iniciativa.

Os resultados da validação deste terceiro modelo lógico estão consolidados na Tabela 1, incluindo as apostas formuladas para cada ação do modelo; as condições de invalidação, a probabilidade de ocorrerem, o impacto e a vulnerabilidade associados; e as ações preventivas ou de controle formuladas para essas condições. Essa análise aplica-se à verificação de eventuais pontos frágeis das ações das PDP que afetariam o alcance dos resultados pretendidos.

\section{Tabela 1}

Teste de consistência e análise de vulnerabilidade das Parcerias para o Desenvolvimento Produtivo (PDP)

\begin{tabular}{|c|c|c|c|}
\hline \multicolumn{4}{|c|}{$\begin{array}{l}\text { Ação } 1 \text { do modelo: Análise de produtos estratégicos para o Sistema Único de Saúde (SUS) } \\
\text { Ação } 2 \text { do modelo: Fomento para apresentação de propostas de projeto de PDP } \\
\text { Aposta: Se forem identificados os produtos estratégicos para o SUS e for publicada a respectiva lista para fomentar a apresentação de } \\
\text { propostas de projeto de PDP, terão produtores públicos e entidades privadas interessadas no estabelecimento de parcerias. A menos que: }\end{array}$} \\
\hline Condições de invalidação & Probabilidade & Impacto & Vulnerabilidade \\
\hline $\begin{array}{l}\text { 1. A qualidade da lista de prioridades em termos de necessidades de saúde não } \\
\text { seja adequada. }\end{array}$ & Média & Alto & Sim \\
\hline \multicolumn{4}{|c|}{$\begin{array}{l}\text { Prevenção e Controle: Uso de matriz multicritério para priorização considerando magnitude, transcendência dos agravos e outros critérios } \\
\text { de relevância. }\end{array}$} \\
\hline $\begin{array}{l}\text { 2. A qualidade da lista de prioridades em termos de prospecção tecnológica, } \\
\text { rastreamento de tecnologias novas e emergentes não seja adequada. }\end{array}$ & Média & Alto & Sim \\
\hline
\end{tabular}




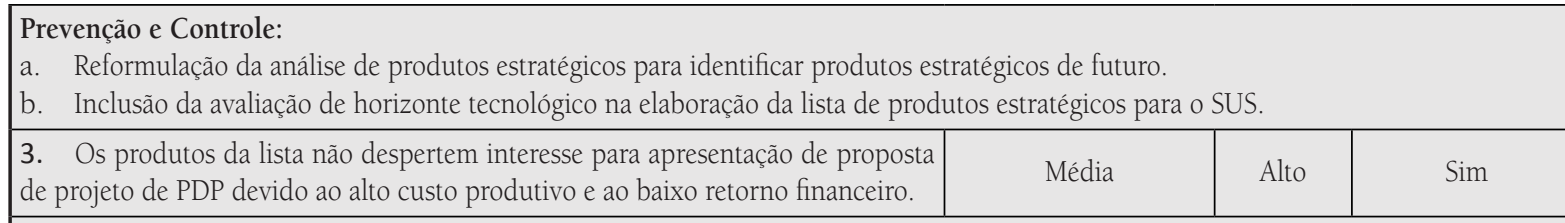

Prevenção e Controle: Priorizar propostas distintas de projetos de PDP, apresentadas pelos mesmos parceiros, que envolvam produtos de alto valor e produtos para doenças e populações negligenciadas.

Ação 3 do modelo: Apresentação de propostas de projeto de PDP

Aposta: Se houver apresentação de propostas de projeto de PDP, então a rede de atores do Complexo Econômico Industrial da Saúde (CEIS) (entidades desenvolvedoras e produtoras públicas e privadas) terá se articulado, fomentando o intercâmbio de conhecimentos para a inovação. A menos que:

\begin{tabular}{l|c|c|c|}
\hline Condição de invalidação & Probabilidade & Impacto & Vulnerabilidade \\
\hline 1. Empresas inidôneas se associem aos produtores públicos. & Média & Alto & Sim \\
\hline Prevenção e Controle: & \\
a. Implementação de processo seletivo de parceiros privados pelos produtores públicos. \\
b. Estabelecimento de critérios para seleção de parceiros pelos produtores públicos. \\
c. Verificação de documentação e pesquisa de relações de mercado do parceiro durante análise do projeto. \\
\hline
\end{tabular}

Ação 4 do modelo: Avaliação de propostas de projeto de PDP

Ação 5 do modelo: Celebração dos compromissos da PDP

Aposta: Se as propostas de projeto de PDP forem avaliadas com parecer favorável e os Termos de Compromisso forem celebrados, os produtores públicos serão responsabilizados pelo investimento, desenvolvimento, transferência e absorção de tecnologia no Brasil, e o Ministério da Saúde (MS) pela aquisição do produto. A menos que:

\begin{tabular}{|l|c|c|c|}
\hline Condição de invalidação & Probabilidade & Impacto & Vulnerabilidade \\
\hline $\begin{array}{l}\text { 1. A atribuição da pontuação da análise da proposta não esteja em conformida- } \\
\text { de com os critérios estabelecidos. }\end{array}$ & Baixa & Alto & Sim \\
\hline
\end{tabular}

\section{Prevenção e Controle:}

a. Cumprimento do processo de análise e decisório estabelecido no marco normativo.

b. Avaliação transparente pela Comissão Técnica e pelo Comitê Deliberativo segundo critérios de análise de mérito e de desempate.

Ação 6 do modelo: Acordo de transferência de tecnologia

Aposta: Se os acordos ou contratos de transferência de tecnologia forem firmados, as condições de transferência e absorção da tecnologia entre os parceiros estarão estabelecidas, bem como a negociação e a gestão de conflitos serão promovidas. A menos que:

\begin{tabular}{|l|c|c|c|}
\hline Condições de invalidação & Probabilidade & Impacto & Vulnerabilidade \\
\hline $\begin{array}{l}\text { 1. A contratualização entre parceiros não esteja em conformidade com a Por- } \\
\text { taria } \mathrm{n}^{\circ} 2531 / 14 \text {, a Lei no } 8666 / 73 \text { e demais normativas e legislação pertinentes. }\end{array}$ & Média & Alto & Sim \\
\hline
\end{tabular}

\section{Prevenção e Controle:}

a. Avaliação dos acordos ou contratos de transferência de tecnologia pela consultoria jurídica dos produtores públicos em conjunto com a equipe técnica responsável pela execução do projeto.

b. Averbação do contrato de transferência de tecnologia no Instituto Nacional de Propriedade Intelectual.

c. Consulta ao MS e órgãos relacionados para dirimir dúvidas.

d. Estabelecimento do contrato conforme condições do Termo de Compromisso.

2. Ingerências políticas sobre os produtores públicos e mudanças no setor governamental ocorram.

Média Alto Sim

\section{Prevenção e Controle:}

a. Forças de associações políticas nos setores legislativo e judiciário.

b. Documentação protocolada de todo o processo administrativo para supervisão dos órgãos de controle.

Ação 7 do modelo: Desenvolvimento do medicamento

Aposta: Se o desenvolvimento do medicamento for concluído satisfatoriamente com registro sanitário pela entidade privada e pelo produtor público, o intercâmbio de conhecimentos terá sido promovido, aumentando a capacidade produtiva e de inovação do país. A menos que:

\begin{tabular}{|c|c|c|c|}
\hline Condições de invalidação & Probabilidade & Impacto & Vulnerabilidade \\
\hline 1. A interação entre parceiros no desenvolvimento não seja efetiva. & Média & Médio & Sim \\
\hline \multicolumn{4}{|c|}{$\begin{array}{l}\text { Prevenção e Controle: } \\
\text { a. Promoção do codesenvolvimento por produtores públicos e entidades privadas. } \\
\text { b. Estabelecimento de cronogramas de treinamento nas entidades privadas para participação da equipe técnica dos produtores públicos. }\end{array}$} \\
\hline 2. O tempo gasto versus o horizonte tecnológico inviabilize o projeto. & Média & Alto & Sim \\
\hline
\end{tabular}




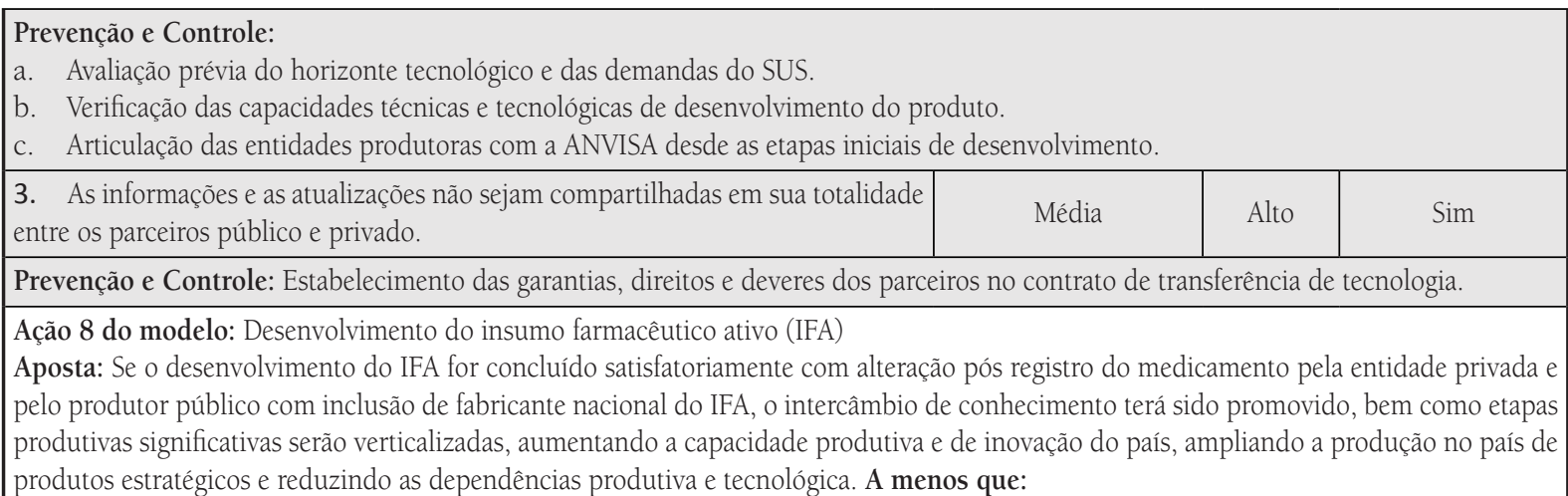

\begin{tabular}{|l|c|c|c|}
\hline Condição de invalidação & Probabilidade & Impacto & Vulnerabilidade \\
\hline $\begin{array}{l}\text { 1. O acesso ao banco mestre de células, no caso de produtos biológicos, e ao } \\
\text { arquivo mestre da droga, no caso de produtos de síntese química, não seja garan- } \\
\text { tido ao produtor público. }\end{array}$ & Média & Alto & Sim \\
\hline
\end{tabular}

\section{Prevenção e Controle:}

a. Estabelecimento das garantias e direitos de uso da tecnologia no contrato de transferência de tecnologia.

b. Fiscalização dos órgãos de fomento.

Ação 9 do modelo: Transferência de tecnologia de produção

Ação 10 do modelo: Transferência de tecnologia de metodologia analítica e controle de qualidade

Aposta: Se as tecnologias de produção, de metodologia analítica e controle de qualidade forem transferidas pelas entidades privadas, serão internalizadas pelos produtores públicos, bem como seus recursos humanos serão capacitados, aumentando a capacidade produtiva e de inovação do país, ampliando a produção no país de produtos estratégicos e reduzindo as dependências produtiva e tecnológica. A menos que:

\begin{tabular}{|l|c|c|c|}
\hline Condições de invalidação & Probabilidade & Impacto & Vulnerabilidade \\
\hline 1. O projeto executivo não seja factível à realidade de execução do produtor público. & Média & Alto & Sim \\
\hline
\end{tabular}

\section{Prevenção e Controle:}

a. Estabelecimento de condicionantes adequadas ao projeto e ao produtor público e de sanções em caso de não evolução da transferência de tecnologia sem justificativas fundamentadas.

\begin{tabular}{|c|c|c|c|}
\hline 2. O planejamento não tenha sido adequado. & Média & Alto & Sim \\
\hline \multicolumn{4}{|c|}{$\begin{array}{l}\text { Prevenção e Controle: } \\
\text { a. Elaboração de projeto executivo conforme orientativo do MS e condições normativas estabelecidas. } \\
\text { b. Verificação de peculiaridades do produtor público ou da tecnologia a ser transferida para adaptação das atividades. }\end{array}$} \\
\hline As tecnologias transferidas pelo parceiro privado sejam obsoletas. & Média & Alto & Sim \\
\hline
\end{tabular}

\section{Prevenção e Controle: \\ a. Análise das tecnologias previstas para transferência no projeto executivo e verificação das efetivamente transferidas. \\ b. Verificação prévia do horizonte tecnológico.}

\begin{tabular}{|l|l|l|l|}
\hline 4. A tecnologia não seja implementada efetivamente em cada etapa. & Alta & Alto & Sim \\
\hline
\end{tabular}

\section{Prevenção e Controle:}

a. Transferência de tecnologia de forma reversa: 1) embalagem secundária, 2) embalagem primária e 3) formulação. Implementação efetiva de cada etapa produtiva no produtor público.

b. Em cada etapa, treinamento e capacitação de recursos humanos do produtor público na entidade privada, acompanhamento in loco do processo, seguido de estabelecimento de procedimento operacional padrão no produtor público, qualificação de equipamento e validação de processo, petição de alteração pós registro na ANVISA, análise ANVISA e publicação do deferimento.

c. Compartilhamento de métodos analíticos, especificações de excipientes, IFA e produto acabado. Execução de testes, elaboração e aprovação de relatórios de covalidação, elaboração e aprovação de protocolos.

d. Implementação efetiva do controle de qualidade no produtor público, realização de testes para liberação de lotes produzidos do medicamento e lotes utilizados do IFA.

\section{Ação 11 do modelo: Adequação fabril}

Aposta: Se forem promovidas adequações nas instalações produtivas dos produtores públicos e obtidos os Certificados de Boas Práticas de Fabricação, os processos de qualificação e validação serão aprimorados e os produtores públicos estarão capacitados produtiva e tecnologicamente, aumentando a capacidade produtiva e de inovação do país, ampliando a produção no país de produtos estratégicos e reduzindo as dependências produtiva e tecnológica. A menos que:

\begin{tabular}{|l|c|c|c|}
\hline Condições de invalidação & Probabilidade & Impacto & Vulnerabilidade \\
\hline $\begin{array}{l}\text { 1. O planejamento de novas áreas produtivas ou reformas seja inadequado, sem } \\
\text { previsão, por exemplo, de tempo de análise de projetos de obras nas Vigilâncias } \\
\text { Sanitárias (VISA) locais ou da fonte de recursos. }\end{array}$ & Alta & Alto & Sim \\
\hline
\end{tabular}




\section{Prevenção e Controle:}

a. Para obras: elaboração de projeto conceitual, projeto básico, plantas baixas, submissão na VISA, análise, exigências, respostas, aprovação, elaboração de edital, licitação e execução.

b. Para equipamentos: elaboração de especificação técnica, licitação, aquisição, entrega e instalação.

2. A execução e implementação de projetos objeto de convênios e outros instrumentos para aquisição de equipamentos e reformas pelos produtores públicos não esteja conforme os requisitos do Fundo Nacional de Saúde (FNS).

Média

\begin{tabular}{l|l|} 
Alto & Sim \\
\hline
\end{tabular}

\section{Prevenção e Controle:}

a. Articulação entre FNS, Secretaria de Ciência, Tecnologia e Insumos Estratégicos (SCTIE) do MS e produtor público por meio de reuniões e visitas técnicas.

b. Realização de oficinas orientativas pelo FNS.

3. As reformas e adequações de áreas produtivas dos produtores públicos não sigam os requisitos sanitários.

\section{Prevenção e Controle:}

a. Interação entre VISA locais, ANVISA e produtores públicos durante as etapas de planejamento e execução de reformas e adequações.

b. Apoio das entidades parceiras aos produtores públicos.

Ação 12 do modelo: Aquisição de produto pelo MS

Aposta: Se o medicamento for produzido pelos parceiros da PDP e adquirido pelo MS conforme os princípios de economicidade e vantajosidade, a oferta do medicamento será ampliada, atendendo as necessidades de saúde da população brasileira e racionalizando o poder de compra do Estado. A menos que:

\begin{tabular}{|c|c|c|c|}
\hline Condições de invalidação & Probabilidade & Impacto & Vulnerabilidade \\
\hline $\begin{array}{l}\text { 1. O contrato entre MS e produtor público não esteja em conformidade com os } \\
\text { critérios e orientações da Portaria no } 2531 / 14 \text {, da Lei no } 8666 / 73 \text {, demais norma- } \\
\text { tivas e legislação pertinente. }\end{array}$ & Média & Alto & Sim \\
\hline
\end{tabular}

\section{Prevenção e Controle:}

a. Negociações prévias entre MS e produtor público.

b. Articulação entre secretarias e departamentos do MS responsáveis pela demanda do produto, pela aquisição e pelo monitoramento dos projetos de PDP.

c. Diagnóstico atualizado da capacidade dos produtores públicos.

2. Práticas de dumping no mercado inviabilizem o fornecimento do produto pela PDP.

Média

\begin{tabular}{|l|l}
\hline Alto & Sim
\end{tabular}

\section{Prevenção e Controle:}

a. Acompanhamento das práticas de mercado antes e durante a implementação do projeto.

b. Acionamento de órgãos de controle pelas entidades produtoras para verificação das práticas.

c. Atuação dos produtores junto ao Conselho Administrativo de Defesa Econômica (CADE).

3. Os recursos obtidos sejam incompatíveis com as necessidades de investimentos.

Baixa

Sim

Prevenção e Controle: Planejamento dos recursos necessários e verificação prévia de fontes de financiamento necessárias.

\section{Ação 13 do modelo: Monitoramento}

Aposta: Se o monitoramento das PDP for realizado pelo MS e ANVISA no âmbito dos Comitês Técnico Regulatórios (CTR), e pelos próprios parceiros das PDP, aumenta-se a probabilidade de alcance dos resultados e de sucesso das PDP. A menos que:

\begin{tabular}{|l|c|c|c|}
\hline Condições de invalidação & Probabilidade & Impacto & Vulnerabilidade \\
\hline $\begin{array}{l}\text { 1. Recursos humanos ou financeiros sejam insuficientes para execução das ati- } \\
\text { vidades de monitoramento. }\end{array}$ & Alta & Médio & Sim \\
\hline
\end{tabular}

\section{Prevenção e Controle:}

a. Articulação do MS com o Ministério do Planejamento, Desenvolvimento e Gestão para realização de concurso público.

b. Articulação do MS com outros órgãos ministeriais e agências (como Ministério da Indústria, Comércio Exterior e Serviços - MDIC;

Ministério da Ciência, Tecnologia, Inovações e Comunicações - MCTIC; e ANVISA) para apoio às atividades de monitoramento.

c. Criação de instrumentos e metodologias de monitoramento e disponibilização de recursos para essa ação.

2. Os instrumentos utilizados não estejam adequados aos projetos e não permitam o controle e correções em tempo oportuno.

\section{Prevenção e Controle:}

a. Adequação dos instrumentos e das metodologias de monitoramento aos diferentes tipos de projeto e às realidades dos produtores públicos.

b. Articulação do MS com órgãos de controle para verificação dos instrumentos e processos implementados.

c. Realização de auditorias de controle interno. 


\begin{tabular}{|c|c|c|c|}
\hline \multicolumn{4}{|c|}{$\begin{array}{l}\text { Ação } 14 \text { do modelo: Internalização completa } \\
\text { Aposta: Se a tecnologia for internalizada pelo produtor público em condições de portabilidade tecnológica, as dependências produtiva e } \\
\text { tecnológica serão reduzidas e o acesso da população a produtos estratégicos será ampliado. A menos que: }\end{array}$} \\
\hline Condições de invalidação & Probabilidade & Impacto & Vulnerabilidade \\
\hline 1. A entidade privada não esteja preparada para a transferência de tecnologia. & Média & Alto & Sim \\
\hline \multicolumn{4}{|c|}{$\begin{array}{l}\text { Prevenção e Controle: } \\
\text { a. Verificação prévia do histórico, capacidade produtiva e tecnologia da entidade privada. } \\
\text { b. Acompanhamento e monitoramento efetivo de todas as etapas de transferência e absorção de tecnologia. }\end{array}$} \\
\hline 2. O produtor público não absorva totalmente a tecnologia. & Média & Alto & Sim \\
\hline \multicolumn{4}{|c|}{$\begin{array}{l}\text { Prevenção e Controle: } \\
\text { a. Planejamento de todas as atividades conforme capacidade do produtor público. } \\
\text { b. Acompanhamento e monitoramento efetivo de todas as etapas de transferência e absorção de tecnologia. }\end{array}$} \\
\hline
\end{tabular}

Fonte: elaboração própria (2016).

A atividade de transferência de tecnologia é complexa e seu sucesso depende de uma série de fatores: capacidades de absorção e gerenciais, modos de transferência e habilidade de desenvolvimento de capacidades tecnológicas ${ }^{20}$.
Dessa forma, o estabelecimento de indicadores, tanto de monitoramento quanto de avaliação, é importante para controle das PDP. Estes indicadores foram elaborados com base no terceiro modelo lógico e estão demonstrados na Tabela 2.

\section{Tabela 2}

Indicadores de monitoramento e avaliação das Parcerias para o Desenvolvimento Produtivo (PDP)

\begin{tabular}{|c|}
\hline Denominação \\
\hline $\begin{array}{c}\text { Taxa de } \\
\text { competitividade por } \\
\text { tecnologia }\end{array}$ \\
\hline $\begin{array}{c}\text { Taxa de aprovação de } \\
\text { propostas de PDP }\end{array}$ \\
\hline $\begin{array}{l}\text { Efetividade das ações } \\
\text { do projeto }\end{array}$ \\
\hline $\begin{array}{c}\text { Otimização das ações } \\
\text { do projeto }\end{array}$ \\
\hline $\begin{array}{c}\text { Proporção de } \\
\text { produtores com } \\
\text { Certificado de Boas } \\
\text { Práticas de Fabricação } \\
\text { (CBPF) vigente }\end{array}$ \\
\hline $\begin{array}{c}\text { Aplicação do poder de } \\
\text { compra da saúde nas } \\
\text { PDP }\end{array}$ \\
\hline
\end{tabular}

\section{Conceito \\ $\mathrm{N}^{\circ}$ de propostas recebidas por tecnologia ou produto no ano} Indicadores de produtos (Indicadores de monitoramento) considerado.

Proporção de propostas de projeto de PDP aprovadas quanto ao mérito em relação ao total de propostas recebidas.

Proporção de PDP na fase "N" com ação "x" concluída, gerando produto " $y$ ".

Percentual de projetos executados dentro do prazo estabelecido nos projetos segundo o número total de projetos aprovados por produtor público, no ano considerado.

Percentual de produtores participantes de PDP com CBPF para a linha produtiva do produto objeto de PDP.

Percentual das aquisições totais do MS que corresponde a aquisições de produtos de PDP no ano considerado.
$\mathrm{N}^{\mathrm{o}}$ total de propostas recebidas no ano considerado para cada tecnologia ou produto da lista de produtos estratégicos

$\mathrm{N}^{\circ}$ de propostas de projetos de PDP aprovadas quanto ao mérito no ano considerado/ $\mathrm{N}^{\circ}$ total de propostas recebidas

$\mathrm{N}^{\circ}$ de PDP na fase "N" com ação " $\mathrm{x}$ " concluída e produto " $\mathrm{y}$ " gerado/ $\mathrm{N}^{\circ}$ total de PDP na fase "N"

( $\mathrm{N}^{\circ}$ de projetos executados dentro do cronograma estabelecido no projeto executivo por produtor público/ $\mathrm{N}^{\circ}$ total de projetos aprovados por produtor público) x 100

$\left(\mathrm{N}^{\circ}\right.$ de produtores participantes de PDP com CBPF vigente para a linha produtiva do produto objeto de PDP/ $\mathrm{N}^{\circ}$ total de produtores com propostas aprovadas de PDP) X 100

(Valor investido pelo MS na aquisição de produtos das PDP no ano considerado/Valor total investido pelo MS no financiamento de produtos) $\mathrm{x} 100$
Fontes de verificação

Sistema Integrado de Protocolo e Arquivo (SIPAR), portal do MS

SIPAR, portal MS

Relatório de acompanhamento, visita técnica, reunião do CTR

Projeto executivo,

Relatório de

acompanhamento, visita técnica, reunião do CTR

Portal ANVISA, Portal MS

DOU e portal MS 


\begin{tabular}{|c|c|c|c|}
\hline Denominação & Conceito & Método de cálculo & Fontes de verificação \\
\hline \multirow[t]{2}{*}{$\begin{array}{c}\text { Proporção de projetos } \\
\text { concluídos no âmbito } \\
\text { das PDP }\end{array}$} & $\begin{array}{l}\text { Distribuição percentual de } \\
\text { projetos concluídos no âmbito } \\
\text { das PDP segundo o número total } \\
\text { de projetos aprovados no ano } \\
\text { considerado. }\end{array}$ & $\begin{array}{l}\text { (No de PDP em fase IV no ano } \\
\text { considerado/ } \mathrm{N}^{\circ} \text { total de PDP } \\
\text { aprovadas) } \times 100\end{array}$ & $\begin{array}{c}\text { Relatório de } \\
\text { acompanhamento, } \\
\text { visita técnica, reunião } \\
\text { do CTR }\end{array}$ \\
\hline & \multicolumn{3}{|c|}{ Indicadores de resultados intermediários (Indicadores de avaliação) } \\
\hline $\begin{array}{l}\text { Índice de adesão de } \\
\text { produtores públicos } \\
\text { às PDP }\end{array}$ & $\begin{array}{l}\text { Índice de produtores públicos } \\
\text { que apresentaram propostas no } \\
\text { ano considerado. }\end{array}$ & $\begin{array}{l}\mathrm{N}^{\circ} \text { de produtores públicos que } \\
\text { apresentaram propostas no ano } \\
\text { considerado/ } \mathrm{N}^{\circ} \text { total de produtores } \\
\text { públicos }\end{array}$ & DOU e portal MS \\
\hline $\begin{array}{l}\text { Adesão de entidades } \\
\text { privadas às PDP }\end{array}$ & $\begin{array}{l}\mathrm{N}^{\circ} \text { de entidades privadas em } \\
\text { propostas apresentadas no ano } \\
\text { considerado. }\end{array}$ & $\begin{array}{l}\mathrm{N}^{\circ} \text { de entidades privadas em } \\
\text { propostas apresentadas no ano } \\
\text { considerado. }\end{array}$ & DOU e portal MS \\
\hline $\begin{array}{c}\text { Taxa de rejeição de } \\
\text { produtos estratégicos }\end{array}$ & $\begin{array}{l}\text { Taxa de produtos da lista que } \\
\text { não foram objeto de propostas } \\
\text { de projeto de PDP no ano } \\
\text { considerado. }\end{array}$ & $\begin{array}{l}\mathrm{N}^{0} \text { de produtos que não foram objeto } \\
\text { de propostas no ano considerado/ } \\
\mathrm{N}^{\circ} \text { total de produtos da lista de } \\
\text { estratégicos }\end{array}$ & DOU e portal MS \\
\hline $\begin{array}{l}\text { Cobertura da RENAME } \\
\text { pela lista de produtos } \\
\text { estratégicos }\end{array}$ & $\begin{array}{l}\text { Percentual de produtos na lista } \\
\text { de produtos estratégicos em } \\
\text { razão dos produtos constantes na } \\
\text { RENAME no ano considerado. }\end{array}$ & $\begin{array}{l}\text { ( } \mathrm{N}^{\mathrm{o}} \text { de produtos na lista de } \\
\text { estratégicos no ano considerado/ } \mathrm{N}^{\mathrm{o}} \\
\text { de produtos da RENAME) x } 100\end{array}$ & DOU e portal MS \\
\hline $\begin{array}{l}\text { Evolução da oferta de } \\
\text { medicamentos }\end{array}$ & $\begin{array}{l}\text { Proporção de unidades } \\
\text { farmacêuticas ofertadas em } \\
\text { relação ao ano anterior }\end{array}$ & $\begin{array}{l}\mathrm{N}^{o} \text { de unidades farmacêuticas do } \\
\text { produto objeto de PDP ofertadas } \\
\text { no ano considerado/ } \mathrm{N}^{\circ} \text { de unidades } \\
\text { farmacêuticas ofertadas no ano } \\
\text { anterior }\end{array}$ & DOU e portal MS \\
\hline $\begin{array}{c}\text { Economia do MS com } \\
\text { as PDP }\end{array}$ & $\begin{array}{l}\text { Diferença de investimentos do } \\
\text { MS com aquisição de produtos } \\
\text { antes das PDP e com as PDP }\end{array}$ & $\begin{array}{l}\text { Valor gasto pelo MS na aquisição } \\
\text { de produtos antes das PDP (-) Valor } \\
\text { gasto com as PDP }\end{array}$ & DOU e portal MS \\
\hline \multicolumn{4}{|c|}{ Indicador de resultado final (Indicador de avaliação) } \\
\hline $\begin{array}{l}\text { Evolução do acesso a } \\
\text { medicamentos }\end{array}$ & $\begin{array}{l}\text { Percentual de pessoas tratadas } \\
\text { com medicamentos objeto de } \\
\text { PDP em relação ao ano anterior }\end{array}$ & $\begin{array}{l}\text { ( } \mathrm{N}^{o} \text { de pessoas tratadas com o } \\
\text { medicamento objeto de PDP no ano } \\
\text { considerado/No de pessoas tratadas } \\
\text { com o medicamento objeto de PDP } \\
\text { no ano anterior) x } 100\end{array}$ & Relatórios do DAF \\
\hline
\end{tabular}

Siglas: CTR - Comitê Técnico Regulatório, DAF - Departamento de Assistência Farmacêutica e Insumos Estratégicos, DOU - Diário Oficial da União, MS - Ministério da Saúde, RENAME - Relação Nacional de Medicamentos Essenciais.

Fonte: elaboração própria (2016).

Os indicadores para monitoramento envolvem sete indicadores de produtos e servem para verificar o quanto as ações executadas para implementação das PDP têm gerado produtos previstos.

A taxa de competitividade por tecnologia está relacionada aos produtos: tecnologias estratégicas mapeadas e constantes em lista publicada no Diário Oficial da União e projeto com a proposta de PDP protocolado no MS. Com esse indicador, é possível monitorar a qualidade da lista de produtos estratégicos, identificando as tecnologias que devem ser objeto de outras iniciativas.

A taxa de aprovação de propostas relaciona-se à fase I do processo de estabelecimento das parcerias e fornece subsídio para o monitoramento da qualidade dos projetos ao se mensurar quanto das propostas teve aprovação de mérito pelas instâncias de avaliação. Segundo a Portaria $n^{\circ} 2531$, os critérios de mérito consideram o atendimento do projeto às diretrizes da normativa, a consonância dos objetivos às políticas públicas de saúde, a adequação do cronograma à complexidade tecnológica e aos requisitos sanitários, a racionalidade dos investimentos, o respeito à propriedade intelectual, o grau de integração produtiva, a economia prevista para o SUS, o planejamento de capacitações, a relevância da parceria para reduzir a vulnerabilidade do SUS e 
contribuir para o desenvolvimento, a insuficiência de fabricação nacional do produto e a capacidade produtiva e intelectual dos produtores.

Para analisar a efetividade e a otimização das ações do projeto, foram propostos dois indicadores que se referem aos produtos das fases II, de implementação do projeto, e III, de transferência de tecnologia. O primeiro deles relaciona as fases concluídas e a capacidade de realizar as atividades do projeto, requerendo supervisão local nos produtores públicos e privados. O segundo inclui a variável tempo, auxiliando no monitoramento da execução do processo conforme cronograma estabelecido. Para verificação da ação de adequação fabril relacionada a essas fases, foi também proposto o indicador de proporção de produtores que adotam boas práticas de fabricação.

$\mathrm{Na}$ perspectiva econômica, sugeriu-se um indicador que mensura o grau de aplicação do poder de compra do MS nas PDP, considerando os valores investidos na aquisição de produtos objetos da iniciativa e a totalidade investida em medicamentos para a assistência.

Associado à fase IV, de internalização de tecnologia, e ao último produto do modelo lógico, relatório de encerramento, incluiu-se o indicador que analisa a distribuição percentual de projetos concluídos em relação à totalidade de PDP.

Os indicadores para avaliação envolvem seis indicadores de resultados intermediários e um indicador de resultado final e têm o propósito de auxiliar a governança com informações sobre os resultados pretendidos com as PDP (componentes Resultados Intermediários e Final da Figura 3).

Os indicadores de resultados intermediários, índice de adesão de produtores públicos e privados às PDP e taxa de rejeição de produtos estratégicos, auxiliam na mensuração de alcance do resultado "instituições públicas e entidades privadas interessadas no estabelecimento de parcerias". Permite, pois, a avaliação do impacto da lista de produtos estratégicos e da iniciativa de PDP para atrair o setor produtivo a executar projetos de alto risco tecnológico.

Os indicadores que verificam a evolução da oferta de medicamentos e o quanto de medicamentos da Relação Nacional de Medicamentos Essenciais (RENAME) é coberto pela lista de produtos estratégicos estão associados à avaliação do fomento ao desenvolvimento científico e tecnológico, bem como ao atendimento das necessidades de saúde da população brasileira.

O alcance da racionalização do poder de compra do Estado pode ser avaliado por meio do indicador de economia do MS com as PDP, o qual indica a diferença, em termos monetários, entre aquisições do mesmo produto antes e durante a parceria devido à redução anual de preços prevista no contrato de transferência de tecnologia.

Por último, foi proposto um indicador de resultado final que fornece subsídios para avaliar a contribuição das PDP para a ampliação do acesso da população a produtos estratégicos por meio do cálculo do percentual de pessoas tratadas com medicamentos objetos de parcerias em relação ao ano anterior ao projeto.

\section{CONCLUSÃO}

Os modelos lógicos construídos com base no PES demonstraram que as ações das PDP estão organizadas de modo a gerar produtos e resultados. Há objetivos definidos, beneficiários e público alvo. Tais modelos compõem o estudo de avaliabilidade, cujos resultados revelaram que as PDP se encontram normatizadas de modo a permitir a realização de avaliações sistematizadas.

Os modelos lógicos e os indicadores propostos podem auxiliar a governança na avaliação das parcerias com o propósito de verificar o desempenho das PDP e o alcance dos produtos e resultados. Embora ainda possa ser cedo para mensurar o impacto da iniciativa, é possível e recomendável a estruturação de uma avaliação a partir dos modelos construídos, congregando os aspectos econômico e social.

Vale ressaltar, entretanto, que os modelos lógicos são modelos de inteligência estratégica que exigem permanente acompanhamento do ambiente, portanto, devem ser revisados periodicamente, verificando seus pressupostos, uma vez que mudanças podem ocorrer e afetar a estrutura lógica, bem como o desempenho ${ }^{11}$ das PDP.

As ações identificadas para as condições de invalidação podem também subsidiar o gerenciamento das PDP de forma a antever e controlar pontos frágeis que afetariam o processo de transferência e absorção de tecnologia pelos produtores e o atingimento das metas. 
Os indicadores de monitoramento e avaliação formulados dão suporte à tomada de decisão e permitem a sua divulgação em painel no portal eletrônico do MS, podendo, inclusive, ser abrigado em plataformas existentes, a exemplo da Sala de Apoio à Gestão Estratégica do MS. Tal medida corresponde a um sistema de acompanhamento para a sociedade que possibilita a transparência das ações e o almejado controle social. Recomenda-se que, a partir deste estudo, seja conduzida a avaliação da estratégia de forma a verificar os resultados apreendidos e as melhorias necessárias, buscando a sustentação das PDP como política de Estado.

\section{REFERÊNCIAS}

1. Gadelha CAG. O complexo industrial da saúde e a necessidade de um enfoque dinâmico na economia da saúde. Ciênc Saúde Coletiva. 2003;8(2):521-35.

2. Cassiolato JE, Szapiro MHS. Os dilemas da política industrial e de inovação: os problemas da Região Sudeste são os do Brasil. In: Um olhar territorial para o desenvolvimento: Sudeste. Rio de Janeiro: Banco Nacional de Desenvolvimento Econômico e Social. 2015; 284-317.

3. Andrade WW, Perini E. Contribuição dos laboratórios farmacêuticos públicos na política de aquisição de medicamentos do Ministério da Saúde. Assoc Bras Econ Saúde. 2014.

4. Domingues CMAS, Woycicki JR, Rezende KS, Henriques CMP. Programa nacional de imunização: a política de introdução de novas vacinas. Gest E Saúde. 2015;6(4):3250-74.

5. Soares PF. Compreendendo se e como os condicionantes dos laboratórios públicos produtores de imunobiológicos no Brasil interferem na atuação do gestor público [tese]. Universidade Federal do Rio de Janeiro; 2012.
6. Rezende KS. As parcerias para o desenvolvimento produtivo e estímulo à inovação em instituições farmacêuticas públicas e privadas [dissertação]. Escola Nacional de Saúde Pública Sérgio Arouca; 2013.

7. Golgher D, Rodrigues R, Massafera RC. Biosimilars in Brazil: developments in 2015 and business perspectives. J Commer Biotechnol. 2015.

8. Barbosa PMN, Castro RMR. A desnecessidade de licitação nos contratos de transferência de tecnologia nas Parcerias de Desenvolvimento Produtivo. Rev ABPI. 2014;133:43-51.

9. Oliveira CS, Sant'Anna LS, Ferreira AP. Licença compulsória e a parceria de desenvolvimento produtivo: assegurando o direito à saúde no Brasil. Rev Juríd Presidência. 2015;17(112):315-40.

10. Wholey JS, Hatry HP, Newcomer KE. Handbook of Practical Program Evaluation. John Wiley \& Sons; 2010. 768 p.

11. Cassiolato M, Gueresi S. Como elaborar Modelo Lógico: roteiro para formular programas e organizar avaliação. 2010. Disponível em: http://www.ipea. gov.br/portal/images/stories/PDFs/100924_notatec6disoc.pdf. Acessado em 18/out/2015. 
12. Scherer FO, Carlomagno MS. Gestão da inovação na prática: como aplicar conceitos e ferramentas para alavancar a inovação. 2a ed. São Paulo: Atlas; 2016.

13. World Health Organization. Equitable access to essential medicines: a framework for collective action. WHO Policy Perspect Med. 2004

14. Ministério da Saúde (BR). Sala de Apoio a Gestão Estratégica. Gestão/Financiamento: Transferência Direta e Fundo a Fundo. Disponível em: http://sage. saude.gov.br/\#14. Acessado em 11/maio/2016.

15. Massuda A. O papel do Estado: a agenda do Ministério da Saúde na coordenação das ações para inovação, ciência e tecnologia em saúde. In: Seminário Valor Econômico: Indústria Farmacêutica Brasileira - Uma Agenda para Inovação; 2015. Brasília-DF

16. Associação Brasileira da Indústria Farmoquímica e de Insumos Farmacêuticos. Mercado. Disponível em: http://abiquifi.org.br/mercado_/. Acessado em 5/nov/2016.

17. Brasil. Ministério da Saúde. Portaria no 2.531. Redefine as diretrizes e os critérios para a defini- ção da lista de produtos estratégicos para o Sistema Único de Saúde (SUS) e o estabelecimento das Parcerias para o Desenvolvimento Produtivo (PDP) e disciplina os respectivos processos de submissão, instrução, decisão, transferência e absorção de tecnologia, aquisição de produtos estratégicos para o SUS no âmbito das PDP e o respectivo monitoramento e avaliação. Diário Oficial da União. 2014; 13 nov.

18. Sundfeld CA, Souza RP. Parcerias para o desenvolvimento produtivo em medicamentos e a Lei de Licitações. Rev Direito Adm. 2013;264(0):91133.

19. Ministério do Planejamento, Desenvolvimento e Gestão (BR). Plano Plurianual 2016-2019. Disponível em: http://www.planejamento.gov.br/ assuntos/planeja/plano-plurianual. Acessado em 6/ jan/2017.

20. Takahashi VP, Sacomano JB. Proposta de um Modelo Conceitual para Análise do Sucesso de Projetos de Transferência de Tecnologia: estudo em empresas farmacêuticas. Gest Produção. 2002; 9(2):181-200. 